\title{
Docosenoic Acid
}

National Cancer Institute

\section{Source}

National Cancer Institute. Docosenoic Acid. NCI Thesaurus. Code C68352.

A monounsaturated very long-chain fatty acid with a 22-carbon backbone and a single double bond orig inating from either the 3rd, 7th, 9th, 11th or 17th positions from the methyl end. 